\title{
Association Between Subacromial Impingement and Acromiohumeral Distance on MRI
}

\author{
Hee Jin Park, ${ }^{1}$ So Yeon Lee, ${ }^{1,}$ Yoon Jung Choi,, ${ }^{1}$ Jai Hyung Park, ${ }^{2}$ and Eugene Kim ${ }^{2}$ \\ ${ }^{1}$ Department of Radiology, Kangbuk Samsung Hospital, Sungkyunkwan University School of Medicine, Seoul, Republic of Korea \\ ${ }^{2}$ Department of Orthopedic Surgery, Kangbuk Samsung Hospital, Sungkyunkwan University School of Medicine, Seoul, Republic of Korea \\ "Corresponding author: So Yeon Lee, Department of Radiology, Kangbuk Samsung hospital, Sungkyunkwan University School of Medicine, \#108 Pyung-dong, Jongno-gu, Seoul, \\ Republic of Korea. Tel: +82-220011035, Fax: +82-220011030, E-mail: radiology11@hanmail.net
}

Received 2016 December 06; Revised 2017 October 25; Accepted 2017 November 03.

\begin{abstract}
Background: We use MRI to determine the mean acromiohumeral distance(AHD) in subacromial impingement (SI) and to identify which part of the subacromial space has the strongest effect on impingement.

Objectives: To assess the association between AHD and SI and to determine which subacromial site most strongly correlates with impingement.

Patients and Methods: The patient population was composed of 56 (70\%) men and 24 (30\%) women; 45 patients had SI and 35 did not. We measured the shortest distance from the outer margin of the inferior cortex of the acromion to the upper cortex of the humeral head at a total of six points. The mean AHD at each location was compared between patients with and without SI using Mann-Whitney tests. To define the optimal cutoff value at each location for the diagnosis of SI, we applied a receiver operating characteristic (ROC) curve and maximum Youden index.

Results: Mean AHDs in patients with SI were smaller than those of normal patients. Differences in the length of the lateral and central portions were statistically significant $(\mathrm{P}<0.05)$. A 7.9-mm cutoff value at the lateral anterior portion showed $96 \%$ sensitivity and $43 \%$ specificity for SI. Cutoff values of $7.8 \mathrm{~mm}$ (reader 1, senior radiologist) and $7.5 \mathrm{~mm}$ (reader 2, junior radiologist) at the lateral posterior portion showed $89 \%$ and $78 \%$ sensitivity and $46 \%$ and $57 \%$ specificity, respectively.

Conclusion: Patients with SI had narrower AHDs than patients without impingement, and specifically, the lateral and central portions of the acromion played an important role in the development of SI.
\end{abstract}

Keywords: MRI, Shoulder, Impingement, Acromion, Supraspinatus Tendon

\section{Background}

Subacromial impingement (SI) is a spectrum of subacromial space pathologies including partial-thickness supraspinatus tendon tear, tendinosis and calcific tendinitis (1). SI is a very common disorder of the shoulder, accounting for $44 \%-45 \%$ of all shoulder pain $(2,3)$. The subacromial space is defined by the humeral head inferiorly, the anterior border and undersurface of the anterior acromion, the coracoacromial ligament and the acromoclavicular joint superiorly. The distance between the acromion and the humeral head ranges from 1 to $1.5 \mathrm{~cm}$ in healthy subjects, as seen on plain radiography (2). Other studies have reported that the distance ranges from 0.6 to $1.4 \mathrm{~cm}$ in the neutral position (4). The supraspinatus tendon, long head of the biceps tendon, subdeltoid and subacromial bursa are interposed between the acromion and the humeral head (2). The coracoacromial ligament, a very hard structure, impinges the cuff, and is the upper main structure of the subacromial space. Any abnormality that disturbs these anatomic relationships may lead to impingement (5).

Orthopedic surgeons use acromiohumeral distance (AHD) as well as the morphology of the acromion for evaluation of the rotator cuff and surgical decision-making (6). Norwood et al. reported that an AHD less than $7 \mathrm{~mm}$ on anteroposterior radiographs suggest a large rotator cuff tear (7). Others reported that narrowing of the AHD is associated with degeneration of the rotator cuff (2). These previous studies were mainly based on plain radiography. The acromion and humeral head are curved and round, which makes accurate evaluation on plain radiography a challenge.

\section{Objectives}

We sought to use MRI to determine the mean value of AHD in impingement and to identify which part of the sub- 
acromial space affects impingement most. Therefore, the purpose of our study was to use MRI to establish a cutoff value for AHD in SI and to determine which site most strongly correlates with impingement.

\section{Patients and Methods}

\subsection{Case Selection}

Two radiologists and a clinician retrospectively evaluated 80 consecutive patients who underwent shoulder MRI. The reason for MRI examination was shoulder pain and impaired mobility. The patient population was composed of 56 (70\%) men and 24 (30\%) women ( $49 \pm 12$ years, range: 18 - 75 years) who underwent MRI at our institution between May 2012 and May 2013. Thirty-seven cases involved the left shoulder, and 43 cases involved the right. The patient who had severe degenerative joint disease of the acromioclavicular joint was excluded. A diagnosis of impingement was made upon positive physical exam findings of Neer's sign and Hawkin's sign, as indicated by pain when physicians elevate or twist the patient's arm (1). One experienced orthopedic surgeon who had fellowship training in shoulder joint surgery and 20 years of experience performed all clinical evaluations and physical examinations. Positive symptoms of SI were pain worsened by overhead shoulder movement, weakness and loss of motion in the affected shoulder. Pain could also be aggravated when the patient lies on the side of the involved shoulder joint. Loss of motion resulted from painful motion during forward elevation of the arm from 60 to 120 degrees (8). Diagnostic criteria were positive MRI findings (partial rotator cuff tear, sub-deltoid bursitis or calcific tendinitis) with at least one of the combined positive clinical manifestations and positive physical examination findings $(9,10)$. MRI diagnosis of SI was categorized as partial tear of the supraspinatus tendon, tendinosis, calcific tendinitis or subdeltoid bursitis. Full-thickness rotator cuff tears were excluded because the correlation between fullthickness supraspinatus tears and AHD is well-established, and the narrowing of the distance is the result of humeral dislocation (11). Visualization of a partial defect in the supraspinatus tendon extending along either the articular or bursal surface was an MRI finding of a partial tear of the supraspinatus tendon (12). If the partially torn tendon was swollen with signal void on every pulse sequence and calcific lesions on plain radiography, calcific tendinitis was diagnosed. The main MRI finding of subdeltoid bursitis was fluid collection between the deltoid muscle and supraspinatus tendon greater than $3 \mathrm{~mm}$ in thickness (13). If no signal change or disruption of the tendon fiber was visible in the tendon and no fluid collection was observed around the tendon, then the MRI diagnosis was no tendon tear or tendinitis. Exclusion criteria included the presence of fractures (1 case), bone tumors (1 case), or full-thickness tears of the supraspinatus tendon (10 cases). This study was approved by our Institutional ethics review board, and the need for informed consent was waived due to the retrospective nature of the study.

\subsection{MRI Parameters}

MRI examinations were performed using the same protocol in a 1.5 T MRI scanner (Intera, Philips, Best, Netherlands) using an 8-channel shoulder coil. The sequences and imaging parameters of the standard protocol for the shoulder joint are summarized in Table 1 . The position of the patients was supine with the arms in a neutral position (no rotation, no abduction, and no flexion). The coronal images were acquired with cuts made parallel to the supraspinatus tendon, which can be seen on the axial images. The sagittal images were obtained with cuts parallel to the articular surface of the glenoid, as seen on the axial images from the lateral margin of the humerus through the scapular neck.

\subsection{Image Analysis}

MRI data were collected electronically with a picture archiving and communication systems (Infinitt 3.0, Seoul, Korea) workstation by two fellowship-trained musculoskeletal radiologists with 12 and 9 years of experience. The radiologists worked independently and were blinded to clinical information and previous radiologic reports. We measured the shortest distance from the outer margin of the inferior cortex of the acromion to the upper cortex of the humeral head at a total of six points. Neer et al. (14) reported that SI results from impingement of the anterior one-third of the acromion. Therefore, we measured the most anterior portion of the acromion and 4 $\mathrm{mm}$ behind it on the oblique sagittal T2-weighted turbo spin-echo (TSE) image assigned to the most lateral portion of the acromion. Then, we measured the points of two 4-mm slices medial from the most lateral portion of the acromion based on the oblique coronal T2-weighted TSE image (Figure 1). We measured a total of six points (Table 2).

\subsection{Statistical Analysis}

Inter-observer agreement between readers was analyzed using intra-class correlation coefficients (ICCs). The mean AHD of each location in patients with and without SI were compared using Mann-Whitney tests. A p value less than 0.05 was considered statistically significant. ICC values less than 0.4 indicated poor reproducibility, ICC values of 0.4 - 0.75 indicated fair or good reproducibility, and ICC values greater than 0.75 indicated excellent reproducibility (15). To define the optimal cutoff values at each location for the diagnosis of SI, we applied a receiver operating characteristic (ROC) curve and maximum Youden index and calculated the area under the curve (AUC). Using that cutoff 


\begin{tabular}{|c|c|c|c|c|c|c|c|}
\hline Parameter & Coro T2 FSE & Coro T2 FS FSE & Coro PD FSE & Sag T1 FSE & Sag T2 FSE & Axial FS PD FSE & Axial T2 FSE \\
\hline TR, msec & $3200-3500$ & $3200-3500$ & 4000 & 520 & $2800-3000$ & 3000 & 3500 \\
\hline TE, msec & $60-90$ & $60-80$ & 30 & 10 & $60-90$ & 30 & 60 \\
\hline Flip angle, ${ }^{\circ}$ & 90 & 90 & 90 & 90 & 90 & 90 & 90 \\
\hline Matrix size & $356 \times 262$ & $356 \times 262$ & $340 \times 240$ & $340 \times 250$ & $356 \times 250$ & $340 \times 250$ & $356 \times 260$ \\
\hline Field of view, cm & 16 & 16 & 16 & 16 & 16 & 16 & 16 \\
\hline Slice thickness, $\mathbf{m m}$ & 3 & 3 & 3 & 4 & 4 & 4 & 4 \\
\hline Interslice gap, mm & 1 & 1 & 1 & 1 & 1 & 1 & 1 \\
\hline Band width, kHz & 290 & 290 & 290 & 370 & 360 & 290 & 290 \\
\hline Echo train length & 15 & 15 & 23 & 5 & 16 & 14 & 15 \\
\hline Signal average & 2 & 2 & 2 & 2 & 2 & 2 & 2 \\
\hline Scan time, min:sec & $2: 48$ & $2: 50$ & $2: 57$ & 2:04 & 2:07 & $2: 30$ & $2: 30$ \\
\hline
\end{tabular}

Abbreviations: Coro, Coronal; FS, Fat saturation; FSE, Fast spine-echo; Sag, Sagittal; PD, Proton density; TE, Echo time; TR, Repetition time.

\begin{tabular}{|c|c|c|c|c|c|c|}
\hline Diagnosis & $\begin{array}{c}\text { Lateral } \\
\text { Anterior }\end{array}$ & $\begin{array}{c}\text { Lateral } \\
\text { Posterior }\end{array}$ & $\begin{array}{l}\text { Central } \\
\text { Anterior }\end{array}$ & $\begin{array}{c}\text { Central } \\
\text { Posterior }\end{array}$ & $\begin{array}{c}\text { Medial } \\
\text { Anterior }\end{array}$ & $\begin{array}{c}\text { Medial } \\
\text { Posterior }\end{array}$ \\
\hline No impingement & $\begin{array}{l}7.76( \pm 1.70) / \\
7.75( \pm 1.70)\end{array}$ & $\begin{array}{l}7.72( \pm 1.53) / \\
7.68( \pm 1.55)\end{array}$ & $\begin{array}{c}8.20( \pm 1.69) / \\
8.17( \pm 1.72)\end{array}$ & $\begin{array}{l}7.89( \pm 1.70) / \\
7.90( \pm 1.72)\end{array}$ & $\begin{array}{c}9.50( \pm 2.03) \mid \\
9.48( \pm 2.03)\end{array}$ & $\begin{array}{c}8.90( \pm 1.85) / \\
8.89( \pm 1.88)\end{array}$ \\
\hline Impingement & $\begin{array}{l}6.74( \pm 0.97) \mid \\
6.74( \pm 0.96)\end{array}$ & $\begin{array}{c}6.88( \pm 1.10) / \\
6.87( \pm 1.10)\end{array}$ & $\begin{array}{c}7.20( \pm 1.23) / \\
7.19( \pm 1.23)\end{array}$ & $\begin{array}{l}7.02( \pm 1.14) \mid \\
7.00( \pm 1.14)\end{array}$ & $\begin{array}{l}8.75( \pm 1.57) \mid \\
8.76( \pm 1.56)\end{array}$ & $\begin{array}{c}8.70( \pm 1.48) / \\
8.71( \pm 1.47)\end{array}$ \\
\hline Pvalue & $0.004 / 0.006$ & $0.009 / 0.013$ & $0.004 / 0.006$ & $0.019 / 0.022$ & $0.102 / 0.118$ & $0.753 / 0.786$ \\
\hline
\end{tabular}

${ }^{\mathrm{a}}$ Reader 1 / reader 2.

${ }^{\mathrm{b}}$ Data in parentheses represent standard deviation.

${ }^{\mathrm{c}}$ Values are expressed as mean $\pm \mathrm{SD}$.

value, the sensitivity, specificity and $95 \%$ confidence interval (CI) were calculated. All analyses were performed with PSAW software version 10.1 (SPSS Inc., Chicago, IL, USA).

\section{Results}

There were 35 cases without SI. The causes of shoulder symptoms in these patients were adhesive capsulitis (13 cases), acromioclavicular arthritis (11 cases), superior labrum anterior posterior (SLAP) lesion (six cases) and labral Bankart lesion (five cases). Thirty-nine patients had partial-thickness tears, four had sub-deltoid bursitis, and two had calcific tendinitis (Figure 2). The mean age of the SI group was $43 \pm 9.9$ years and that of the no impingement group was $55 \pm 10.0$ years. The mean AHDs of patients with and without SI, stratified by location, are presented in Table 2 and Figure 3. The mean AHD of SI patients was about $6.8 \mathrm{~mm}$ in the lateral portion of the acromion and $7.1 \mathrm{~mm}$ in the central portion. The mean length of AHD in SI patients was shorter than that in patients without SI.
The length differences of the lateral and central portions were statistically significant $(0.004<$ P value $<0.022)$. The length differences of the medial portion were not statistically significant. The ICC of each measurement between two readers was from 0.995 to 0.999 , indicating excellent inter-observer agreement (Table 3 ).

Table 4 presents the optimal cutoff values acquired using the ROC curves. A cutoff value of $7.9 \mathrm{~mm}$ at the lateral anterior portion showed $96 \%$ sensitivity and $43 \%$ specificity. Cutoff values of $7.8 \mathrm{~mm}$ (reader 1 , senior radiologist) and $7.5 \mathrm{~mm}$ (reader 2, junior radiologist) at the lateral posterior portion showed $89 \%$ and $78 \%$ sensitivity and $46 \%$ and $57 \%$ specificity, respectively. The cutoff AUC values of the medial portion were not significantly different $(0.108<\mathrm{P}$ value $<0.791)$.

\section{Discussion}

Saupe et al. reported that reduced AHD is associated with supraspinatus tendon tears, with $90 \%$ of patients 

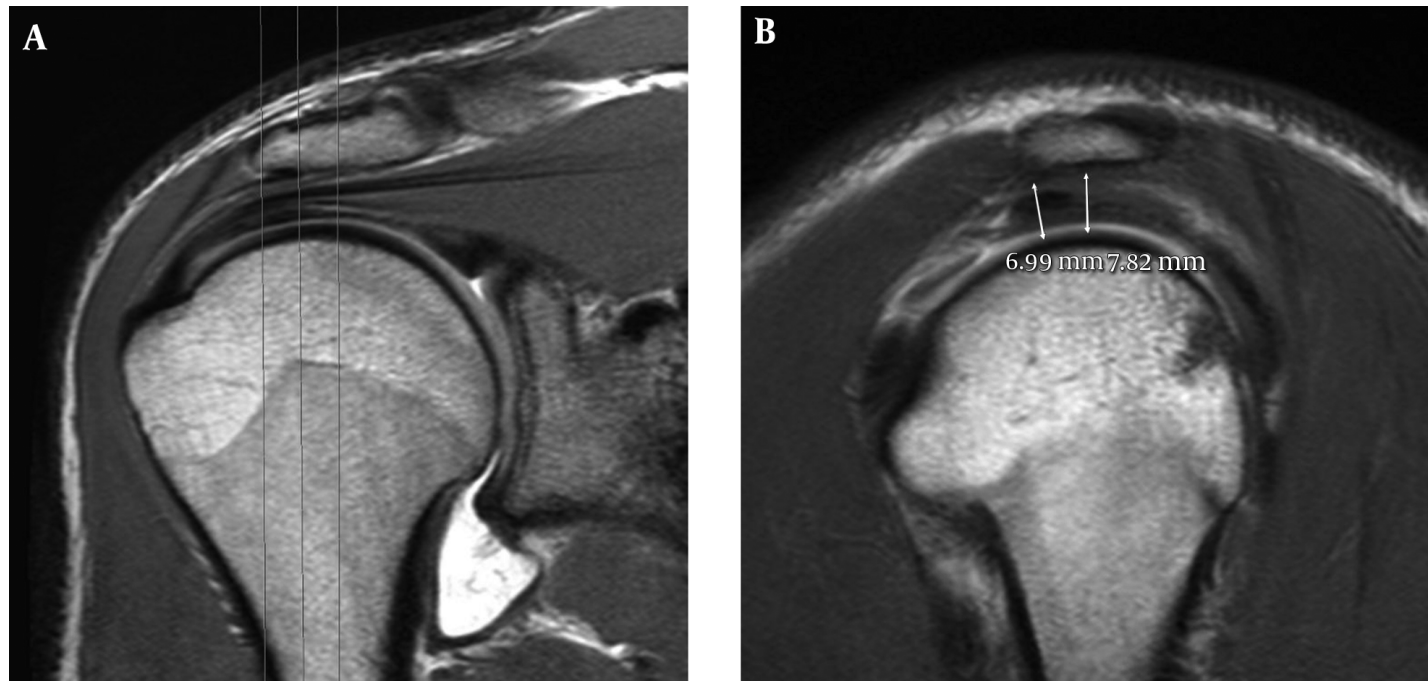

Figure 1. A, The oblique coronal T2-weighted image (repetition time/echo time (TR)/TE), 3200/80) of the shoulder joint at the level of the posterior third of the humeral head shows the most lateral portion of the acromion. Three vertical planes (oblique sagittal view) were used for measurement from the lateral edge of the acromion at $4 \mathrm{~mm}$ intervals. B, The oblique sagittal T2-weighted image (TR/TE, 2900/80) of the shoulder joint. The shortest distance between the inner surface of the acromion and the outer surface of the humeral head is measured from the anterior edge of the acromion and $4 \mathrm{~mm}$ behind (arrows).
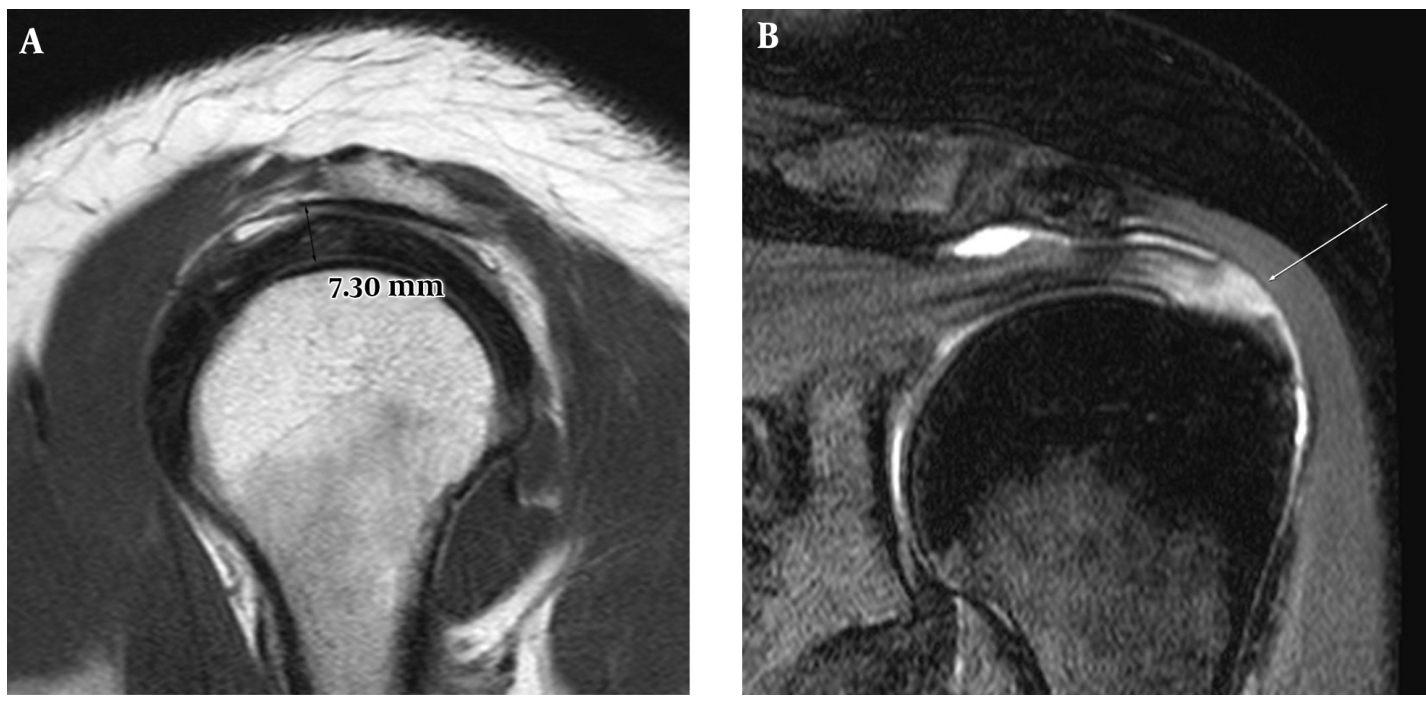

Figure 2. A 36-year-old woman with subacromial impingement syndrome. A, Oblique sagittal T2-weighted image (TR/TE, 2900/80) at the lateral edge of the acromion demonstrates a $7.3 \mathrm{~mm}$ acromiohumeral distance at the anterior portion. B, The oblique fat-suppressed coronal T2-weighted image (TR/TE, 3200/60) shows a partial tear of the supraspinatus tendon (arrow).

\begin{tabular}{lcccccccc}
\hline Table 3. Intra-Class Correlation Coefficients for Distance Measurement Between Readers & & & & \\
\hline Location & Lateral Anterior & Lateral Posterior & Central Anterior & Central Posterior & Medial Anterior & Medial Posterior & P value \\
\hline Value & 0.999 & 0.999 & 0.999 & 0.995 & 0.997 & 0.999 & $<0.001$ \\
\hline
\end{tabular}

with an AHD less than $7 \mathrm{~mm}$ having a full-thickness tear of the supraspinatus tendon (11). The exact location of the measurement on oblique coronal MRI was not described in their study. The optimal cutoff values of our study were $7.5 \mathrm{~mm}$ and $7.8 \mathrm{~mm}$ at the lateral posterior portion, $7.9 \mathrm{~mm}$ at the lateral anterior portion (Figure 2) and 8.8 and 8.1 


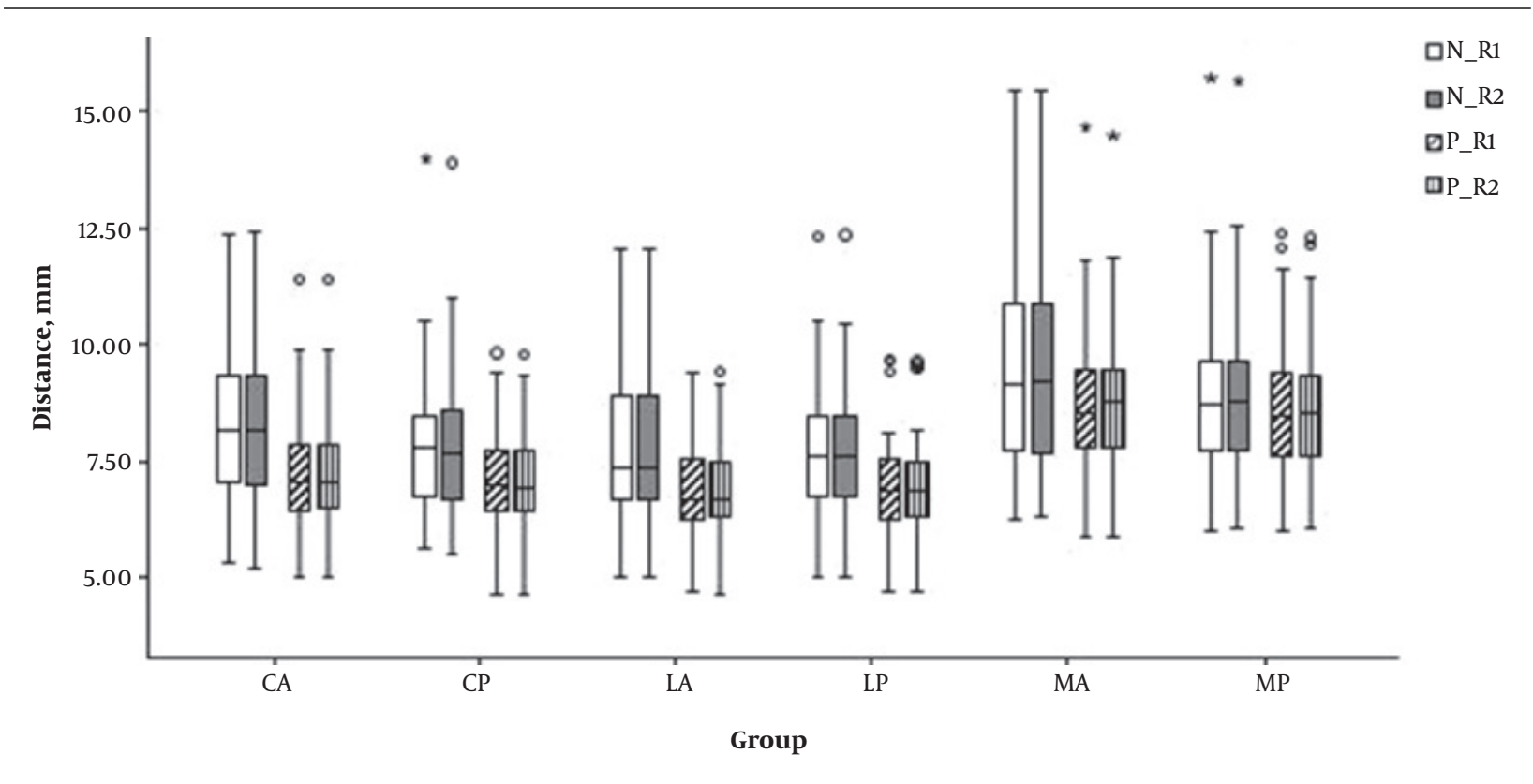

Figure 3. The graph shows the mean acromiohumeral distance according to location. $\mathrm{N}=$ no impingement, $\mathrm{P}=$ subacromial impingement, $\mathrm{R} 1=$ reader 1 , $\mathrm{R} 2=$ reader 2 (Abbreviations: CA, central anterior; CP, central posterior; LA, lateral anterior; LP, lateral posterior ; MA,medical anterior; MP, medical posterior).

\begin{tabular}{|c|c|c|c|c|c|c|}
\hline Location & Optimal Cutoff (mm) & $\begin{array}{l}\text { Area under the ROC } \\
\text { curve and } P \text { value }\end{array}$ & Sensitivity \% & 95\% CI of Sensitivity, \% & Specificity, \% & 95\% CI of Specificity, \% \\
\hline Lateral anterior & $7.91 / 7.89$ & $0.69(0.002) / 0.68(0.004)$ & $95.56 / 95.56$ & $84.9-99.5 / 84.9-99.5$ & $42.86 / 42.86$ & $26.3-60.6 / 26.3-60.6$ \\
\hline Lateral posterior & $7.84 / 7.49$ & $0.67(0.007) / 0.66(0.011)$ & $88.89 / 77.78$ & $75.9-96.3 / 62.9-88.8$ & $45.71 / 57.14$ & $28.8-63.4 / 39.4-73.7$ \\
\hline Central anterior & $8.79 / 8.14$ & $0.69(0.003) / 0.68(0.005)$ & $95.56 / 82.22$ & $84.9-99.5 / 67.9-92.0$ & $42.86 / 54.29$ & $26.3-60.6 / 36.6-71.2$ \\
\hline Central posterior & $7.97 / 7.89$ & $0.65(0.015) / 0.65(0.017)$ & $80.00 / 80.00$ & $65.4-90.4 / 65.4-90.4$ & $48.57 / 45.71$ & $31.4-66.0 / 28.8-63.4$ \\
\hline Medial anterior & $9.69 / 9.65$ & $0.61(0.108) / 0.60(0.125)$ & $80.00 / 80.00$ & $65.4-90.4 / 65.4-90.4$ & $45.71 / 45.71$ & $28.8-63.4 / 28.8-63.4$ \\
\hline Medial posterior & $7.19 / 7.21$ & $0.52(0.758) / 0.52(0.791)$ & $8.89 / 8.89$ & $2.5-21.2 / 2.5-21.2$ & $77.14 / 77.14$ & $59.9-89.6 / 59.9-89.6$ \\
\hline
\end{tabular}

Abbreviations: CI, Confidence interval; ROC, Receiver operating characteristic.

${ }^{\text {a }}$ Reader $1 /$ reader 2

$\mathrm{mm}$ at the central anterior portion of the acromion (Table 4). Although Saupe et al. (11) included full-thickness tears of the supraspinatus in their study, we excluded fullthickness tears because narrowing of the AHD in these cases is already well-established in many reports $(11,16)$. Superior migration of the humeral head is a well-known phenomenon in full-thickness tears of the supraspinatus, and this process is quantified by AHD narrowing. This narrowing results from lack of stabilization by the rotator cuff and the absence of torn tendon structures as space holders (12). Although SI results from abutment between the humeral head and acromion during abduction of the shoulder joint, decreased AHD in the neutral position is also associated with SI. Saupe et al. (11) verified the importance of AHD in the neutral position in the evalua- tion of SI. Our study found that statistically significant differences in AHD exist between patients with and without SI and that the lateral portion of the subacromial space plays a more important role in the development of SI than the medial portion. Anatomical factors that cause narrowing of the AHD include variations in the shape of the acromion, orientation of the slope of the acromion and prominent osseous changes to the inferior aspect of the acromion (17). Some researchers do not agree that the slope of the acromion plays an important role in impingement (18). The acromion is a large, triangular or oblong process, which is flattened in an anterior-posterior direction and projects laterally before curving forward and upward. Neer et al. (19) reported that the anterior third is responsible for characteristic impingement. Proliferative 
spurs and ridges exist on the anterior lip and undersurface of the anterior process of the acromion, and these morphologic characteristics act as therapeutic clues of anterior acromioplasty. In our study, only AHD in the lateral portion of the acromion and $4 \mathrm{~mm}$ medial to that portion were significantly different between groups. These results are mostly in agreement with earlier studies; however, there were no significant differences in AHD between the anterior and posterior portions.

Our study had some limitations. One limitation was the single neutral posture of the shoulder MRI that was evaluated, as a significant change in minimal AHD during abduction has been observed (20). In our study, all MRI procedures were performed in a neutral, supine position, and no abduction was applied. Variation between patients was minimized by using the same position in every patient. Second, this study lacked surgical confirmation of lesions because partial tears of the supraspinatus tendon and calcific tendinitis do not always require surgical intervention. Hence, the diagnostic standard of the reference was imperfect.

In conclusion, SI patients showed narrower AHD than patients without impingement, and the lateral and central portions of the acromion appear to play an important role in the development of SI. AHD on MRI can be a useful ancillary marker for evaluation of SI in patients without a fullthickness tear of the supraspinatus tendon.

\section{Footnotes}

Authors' Contributions: None declared.

Conflict of Interest: We have no conflict of interest to report.

Financial Disclosure: None declared.

Funding/Support: None declared.

\section{References}

1. Koester MC, George MS, Kuhn JE. Shoulder impingement syndrome. Am J Med. 2005;118(5):452-5. doi: 10.1016/j.amjmed.2005.01.040. [PubMed: 15866244].

2. Umer M, Qadir I, Azam M. Subacromial impingement syndrome. Orthop Rev (Pavia). 2012;4(2). e18. doi: 10.4081/or.2012.e18. [PubMed: 22802986].

3. Neer C2. Impingement lesions. Clin Orthop Relat Res. 1983;(173):70-7. doi: 10.1097/00003086-198303000-00010. [PubMed: 6825348].
4. Petersson CJ, Redlund-Johnell I. The subacromial space in normal shoulder radiographs. Acta Orthop Scand. 1984;55(1):57-8. doi: 10.3109/17453678408992312. [PubMed: 6702430].

5. Bigliani LU, Levine WN. Subacromial impingement syndrome. I Bone Joint Surg Am. 1997;79(12):1854-68. doi: 10.2106/00004623-19971200000012. [PubMed: 9409800].

6. Jost B, Pfirrmann CW, Gerber C, Switzerland Z. Clinical outcome after structural failure of rotator cuff repairs. J Bone Joint Surg Am. 2000;82(3):304-14. doi: 10.2106/00004623-200003000-00002. [PubMed: 10724223].

7. Norwood LA, Barrack R, Jacobson KE. Clinical presentation of complete tears of the rotator cuff.J Bone Joint Surg Am.1989;71(4):499-505. doi: 10.2106/00004623-198971040-00004. [PubMed: 2703509].

8. Chen AL, Rokito AS, Zuckerman JD. The role of the acromioclavicular joint in impingement syndrome. Clin Sports Med. 2003;22(2):343-57. doi:10.1016/S0278-5919(03)00015-2. [PubMed: 12825535].

9. Balke M, Bielefeld R, Schmidt C, Dedy N, Liem D. Calcifying tendinitis of the shoulder: midterm results after arthroscopic treatment. Am J Sports Med. 2012;40(3):657-61. doi: 10.1177/0363546511430202. [PubMed: 22156173].

10. Balke M, Banerjee M, Vogler T, Akoto R, Bouillon B, Liem D. Acromial morphology in patients with calcific tendinitis of the shoulder. Knee Surg Sports Traumatol Arthrosc. 2014;22(2):415-21. doi: 10.1007/s00167012-2327-5. [PubMed: 23223878].

11. Saupe N, Pfirrmann CW, Schmid MR, Jost B, Werner CM, Zanetti M. Association between rotator cuff abnormalities and reduced acromiohumeral distance. AJR Am J Roentgenol. 2006;187(2):376-82. doi: 10.2214/AJR.05.0435. [PubMed: 16861541]

12. Magee T, Williams D. 3.0-T MRI of the supraspinatus tendon. AJR Am J Roentgenol. 2006;187(4):881-6. doi: 10.2214/AJR.05.1047. [PubMed: 16985129].

13. Mulyadi E, Harish S, O'Neill J, Rebello R. MRI of impingement syndromes of the shoulder. Clin Radiol. 2009;64(3):307-18. doi: 10.1016/j.crad.2008.08.013. [PubMed:19185661].

14. Neer C2. Anterior acromioplasty for the chronic impingement syndrome in the shoulder: a preliminary report. J Bone Joint Surg Am. 1972;54(1):41-50. doi: 10.2106/00004623-197254010-00003. [PubMed 5054450].

15. Rosner B. Fundamentals of biostatics. 6th ed. Belmont, CA: Duxbury Press; 2005.

16. Weiner DS, Macnab I. Superior migration of the humeral head. A radiological aid in the diagnosis of tears of the rotator cuff. J Bone Joint Surg Br. 1970;52(3):524-7. doi: 10.1302/0301-620X.52B3.524. [PubMed: 5455085].

17. Seitz AL, McClure PW, Finucane S, Boardman N3, Michener LA Mechanisms of rotator cuff tendinopathy: intrinsic, extrinsic, or both? Clin Biomech (Bristol, Avon). 2011;26(1):1-12. doi: 10.1016/j.clinbiomech.2010.08.001. [PubMed: 20846766].

18. Moses DA, Chang EY, Schweitzer ME. The scapuloacromial angle: a 3D analysis of acromial slope and its relationship with shoulder impingement. I Magn Reson Imaging. 2006;24(6):1371-7. doi: 10.1002/jmri.20763. [PubMed: 17075837].

19. Neer C2. Anterior acromioplasty for the chronic impingement syndrome in the shoulder. 1972. J Bone Joint Surg Am. 2005;87(6):1399. doi 10.2106/JBJS.8706.cl. [PubMed: 15930554].

20. Graichen H, Bonel H, Stammberger T, Englmeier KH, Reiser M, Eckstein F. Subacromial space width changes during abduction and rotation-a 3-D MR imaging study. Surg Radiol Anat. 1999;21(1):59-64. [PubMed: 10370995]. 